'This is an author-created, un-copyedited version of an article accepted for publication in Physics in Medicine and Biology.

The publisher is not responsible for any errors or omissions in this version of the manuscript or any version derived from it. The Version of Record is available online at https://doi.org/10.1088/1361-

6560/aa5d3a.'

\title{
Modeling the impact of prostate edema on LDR brachytherapy: a Monte Carlo dosimetry study based on a 3D biphasic finite element biomechanical model
}

\author{
K A Mountris ${ }^{1}$, J Bert ${ }^{1}$, J Noailly ${ }^{2}$, A Rodriguez Aguilera ${ }^{4}$, A Valeri ${ }^{5}$, O Pradier ${ }^{1,6}$, U \\ Schick $^{1,6}$, E Promayon ${ }^{3}$, M A Gonzalez Ballester ${ }^{2,7}$, J Troccaz ${ }^{3}$ and D Visvikis ${ }^{1}$ \\ ${ }^{1}$ LaTIM, INSERM, UMR 1101, CHRU Brest, France \\ ${ }^{2}$ Department of Information and Communication Technologies, Universitat Pompeu \\ Fabra, Barcelona, Spain \\ ${ }^{3}$ Univ. Grenoble Alpes, TIMC-IMAG UMR CNRS 5525, F-38000 Grenoble, France \\ ${ }^{4}$ University of Granada, Spain \\ ${ }^{5}$ Department of Urology, CHRU Brest, France \\ ${ }^{6}$ Department of Radiotherapy, CHRU Brest, France \\ ${ }^{7}$ ICREA, Barcelona, Spain \\ E-mail: konstantinos.mountris@gmail.com
}

\begin{abstract}
Prostate volume changes due to edema occurrence during transperineal permanent brachytherapy should be taken under consideration to ensure optimal dose delivery. Available edema models, based on prostate volume observations, face several limitations. Therefore, patient-specific models need to be developed to accurately account for the impact of edema. In this study we present a biomechanical model developed to reproduce edema resolution patterns documented in the literature. Using the biphasic mixture theory and Finite Element analysis, the proposed model takes into consideration the mechanical properties of the pubic area tissues in the evolution of prostate edema. The model's computed deformations are incorporated in a Monte Carlo simulation to investigate their effect on post-operative dosimetry. The comparison of Day1 and Day30 dosimetry results demonstrates the capability of the proposed model for patient-specific dosimetry improvements, considering the edema dynamics. The proposed model shows excellent ability to reproduce previously described edema resolution patterns and was validated based on previous findings. According to our results, for a prostate volume increase of 10-20\% the Day30 urethra D10 dose metric is higher by $4.2 \%-10.5 \%$ compared to the Day1 value. The introduction of the edema dynamics in Day30 dosimetry shows a significant global dose overestimation identified on the conventional static Day30 dosimetry. In conclusion, the proposed edema biomechanical model can improve the treatment planning of transperineal permanent brachytherapy accounting for postimplant dose alterations during the planning procedure.
\end{abstract}


Keywords: Prostate brachytherapy, edema dynamics, biomechanical model, Monte Carlo simulation, dynamic dosimetry.

\section{Introduction}

Prostate cancer is the third leading cause of cancer deaths in the male population (Haas et al 2008). Trans-rectal ultrasound-guided low-dose rate brachytherapy (LDR) is a widely used low-risk technique for effective prostate cancer treatment. Radioactive seeds of 125I or 103Pd are implanted in the prostate gland, guided by trans-rectal ultrasound imaging in order to deliver a homogeneous tumoricidal dose while sparing the healthy surrounding tissues and organs at risk (Davies et al 2004). The radioactive seeds are implanted in the prostate using needles inserted in the gland through a rectangular grid mounted on the trans-rectal ultrasound probe. Despite the fact that LDR brachytherapy has been established as a routine operation in the last decades, the Brachytherapy Dose Calculation Formalism used in clinical practice is based on the AAPM TG-43 protocol (Rivard et al 2004) and is associated with several limitations. More recent proposals from the AAPM Task Group 186 report provide guidance for the use of alternative and more accurate dose calculation models accounting for non-water equivalent media such as the grid based Boltzmann solver (GBBS) and Monte Carlo (MC) methods (Beaulieu et al 2012). However, in all of these dosimetry approaches, a significant factor of error/uncertainty which is not considered, is the prostate tissue damage due to the implantation process resulting in prostate volume changes during and after the operation. These volume changes, associated with the resulting prostate edema, have a significant effect on the actual dose deposition (Chira et al 2013, Tejwani et al 2012, Leclerc et al 2006 and Waterman et al 1998) to the prostate and the Organs At Risk (OAR).

The initial body response to biological stress, such as with tissue trauma, is an acute inflammation that involves a cascade of events mediated by a large array of cells and molecules that, under normal circumstances, flow freely in the blood circulatory system. The purpose of these cells and molecules is to locate invading pathogens or damaged tissue, eliminate the offending agents, and restore the body homeostasis (Day et al 2006). To maximize the delivery of these mediators in the damaged areas, the capillary permeability increases and blood plasma flows from the vascular network to the interstitial matrix carrying the repairing mediators to their target. This excessive fluid build-up in the interstitial matrix is the main cause of fast occurring edema (Starling 1896), during and after a brachytherapy operation.

Several studies have investigated the influence of edema on post-implant dosimetry (Chira et al 2013, Tejwani et al 2012, Waterman et al 1998, Sloboda et al 2012, Yue et al 2006, Sloboda et al 2010, Chen et al 2000 and Dogan et al 2002). Chira et al (2013) have shown that a prostate volume increase of $10 \%$ during brachytherapy can lead to an increase in D90 (dose coverage of $90 \%$ of the prostate volume) at Day30 of approximately $11.7 \%$ in comparison to the D90 measured at Day1. Leclerc et al (2006) have shown an overall dose increase of up to $15 \mathrm{~Gy}$ when edema was considered in the dosimetry by means of a weighted dose-volume histogram summation algorithm. Based on the same algorithm Villeneuve et al (2008) showed the dose over-estimation in inverse treatment planning systems when treatment planning is done considering pre-implant prostate volumes before the occurrence of edema. In order to improve the post-implant dosimetry, analytical models based on CT and MRI image processing techniques have been developed, considering the edema resolution time course (Tejwani et al 2012, Waterman et al 1998, Sloboda et al 2012 and Sloboda et al 2010, Leclerc et al 2006). The main parameters of these models include the edema magnitude and half-life. In their study, Tejwani et al (2012) have shown an exponential resolution pattern for the edema similarly to previously published studies (Waterman et al 1998). In contrast, Sloboda et al $(2010,2012)$ reported a near-linear edema resolution time course. In these studies, a large variability on 
edema magnitude and half-life parameters amongst patients have been reported. The dependence of this dispersion on parameters such as the Gleason score, the needle gestures, the number of implants, the seed activity and the mechanical properties of the tissue has not been extensively investigated. As such the influence of these parameters is still not clear, therefore they have not been considered in the existing analytical edema models (Nath et al 2009). Additionally, none of these models account for the intraoperative evolution phase of the edema since the dose deposited during the operation is considered negligible (Dogan et al 2002).

The aim of the present study is the improvement of patient-specific edema models, taking into consideration potentially significant parameters that were not considered in previously proposed analytical models. More specifically, we propose a biomechanical computational model based on biphasic mixture theory. The proposed model accounts for the patient-specific geometry of the pubic region and the mechanical properties of the pubic organs of interest. We investigate the impact of the values given to these tissue properties on the edema magnitude, half-life and resolution pattern. The results are compared against clinical findings from relevant studies. These results are finally integrated within a Monte Carlo simulation based dosimetry framework in order to investigate the extent and accuracy of the model's capability to predict relative differences between the estimated deposited dose during treatment planning in Day1 (the day of operation) and that obtained based on Day30 dosimetry findings. The determined relative dose differences in these studies are also compared to related clinical findings.

\section{Materials and Methods}

\subsection{Biomechanical model}

The prostate edema and the associated biomechanical response of the pubic region were modeled using the Finite Element (FE) methodology. Patient specific models were derived from a dataset of pre-operative CT segmented images from 15 patients who underwent LDR brachytherapy within the radiotherapy department of the University Hospital of Brest. Manual segmentation of the prostate and OAR was performed by an experienced clinician. The ISO2MESH toolkit (Fang and Boas 2010) was subsequently employed for the generation of 3D multi-domain tetrahedral mesh representations of the pubic region geometry for each patient. The prostate, bladder, rectum, and the pubic bones were explicitly represented (figure 1).

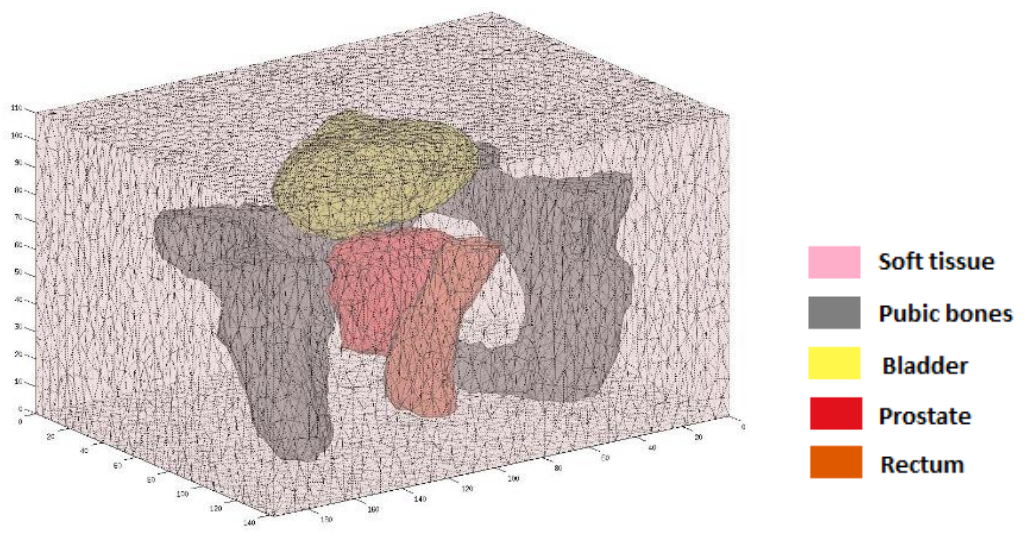


Figure 1. Tetrahedral mesh representation of the pubic area. The organs of interest (prostate, bladder, rectum and pubic bones) are discretized within a soft tissue block approximating the surrounding pubic structures.

The software suite FEBio (Maas et al 2012) was used for the numerical analysis. Biphasic calculations were performed in order to describe the prostate swelling and its effect on the model geometry, similarly to previous studies on brain edema (Li et al 2009) or intervertebral disk swelling (Galbusera et al 2011). More specifically the build-in biphasic mathematical models of FEBio suite (http://febio.org) were used. The edema was treated as a distributed fluid source within the prostate volume representing inflammation and allowing the simulation of fluid accumulation during the operation day (Day1), i.e., the period during which a maximum edema magnitude is reached according to previously published works (Tejwani et al 2012 and Sloboda et al 2010). After Day1 the edema fluid source was "switched off", and the resolution of the edema as a function of time was controlled exclusively by the mechanical and functional parameters assigned to the prostate and the surrounding tissues. According to the biphasic mixture theory, described in (Maas et al 2015), a biphasic material is assumed to be a mixture of a porous permeable solid and an interstitial fluid, both intrinsically incompressible. However, this mixture can change volume as interstitial fluid is exchanged with the porous space of the solid. Under quasi-static conditions the conservation of the mass requires that:

$$
\operatorname{div}\left(\mathbf{v}_{\mathbf{s}}+\mathbf{w}\right)=\mathrm{S}_{\mathrm{w}}
$$

where, $\mathbf{v}_{\mathbf{s}}$ is the solid velocity, $\mathbf{w}$ the fluid flux relative to the solid and $S_{\mathrm{w}}$ is an external fluid supply term. A simplified version of Starling's equation (Starling 1896) was used to simulate the external fluid supply term $S_{\mathrm{w}}$ :

$$
\mathrm{S}_{\mathrm{w}}=\mathrm{k}\left(\mathrm{p}_{\mathrm{v}}-\mathrm{p}\right)
$$

where, $\mathrm{k}$ is the filtration coefficient, $\mathrm{p}_{\mathrm{v}}$ the source pressure that simulates inflammation and $\mathrm{p}$ the mixture's fluid compartment pressure. In the proposed edema model the filtration coefficient $\mathrm{k}$ was assumed equal to 1 (absolute filtration) and the pressure $\mathrm{p}_{\mathrm{v}}$ linearly dependent on time:

$$
p_{v}(t)=\frac{2 \Delta}{k t_{\max }^{2}} t+p
$$

The coefficient multiplying time (t) in (3) was determined so that the total applied external fluid source is equal to the expected edema magnitude (4):

$$
\int_{0}^{t_{\max }} \mathrm{S}_{\mathrm{W}} \mathrm{dt}=\Delta
$$

where, $\Delta$ is the edema magnitude (maximum relative prostate volume increase). The edema magnitude is expressed as:

$$
\Delta=\frac{\mathrm{V}_{\max }-\mathrm{V}_{0}}{\mathrm{~V}_{0}}
$$

where, $V_{\max }$ is the prostate volume associated with maximum edema as measured at Day 1 and $V_{0}$ is the prostate volume with no edema.

A biomechanical model is described by the assigned materials in the different structures and the corresponding material parameters which are described following. The drained solid skeleton of the prostate model was represented as a neo-Hookean hyperelastic material (Wang et al 2016), and the permeability of the saturated biphasic continuum was described through the Holmes-Mow strain-dependent permeability 
model. The rectum and the bladder were considered as nearly incompressible Mooney-Rivlin materials (Haridas et al 2006), and the surrounding continuum (soft tissue in figure 1) was modeled as a compressible neo-Hookean material. Finally, the pubic bones were considered as rigid structures. The mechanical properties assigned to the different structures were derived from previously reported ranges (Ahn et al 2010, Hu et al 2008, Lee et al 2008, Li et al 2014, Barr et al 2012 and Yan et al 2012). In the following computational tests only the mechanical properties of the prostate are assumed to be variable for simplicity. The ranges of the mechanical properties used for the prostate and the fixed values used for the other structures as an input to the proposed biomechanical model are summarized in table 1 . The output of interest from the proposed model were the prostate volume changes computed for a period of 30 days, for a range of given mechanical parameters (Young Modulus, Poisson ratio) and fluid supply source values.

In order to investigate the relationship between the edema behavior (magnitude, half-life) and the prostate elastic parameters (Young modulus, Poisson's ratio) the model's response was evaluated for a range of Young modulus (0.01 - $0.1 \mathrm{MPa})$ and Poisson's ratio $(0.1-0.45)$ values while the other model parameters were maintained constant (Test 1). Additionally, the edema resolution pattern of the model was computed for a mean edema magnitude of $\Delta=0.38$ determined in Tejwani et al (2012). Extreme values of edema magnitude $(\Delta=0.1, \Delta=0.2, \Delta=0.65)$ were also considered using the material parameters derived from the model assessment experiment varying only the external fluid supply (Test 2). Edema magnitude values greater than $\Delta=0.65$ were not considered since they represent clinically unrealistic values and may result in numerical instabilities due to very large deformations. Finally, the edema resolution pattern was computed for the two extreme Young modulus values used in test 1 (i.e., 0.01 and $0.1 \mathrm{MPa}$ ) with the other mechanical properties and external fluid supply set as constants. These findings were compared with the resolution pattern computed using the model assessment parameters (Test 3). The patterns computed in tests 2 and 3 were used to investigate the capabilities of the proposed model to determine the impact of the edema magnitude and the edema resolution pattern on post-implant dosimetry. Table 2 summarizes the different scenarios for which the model's edema resolution pattern was computed for. The edema resolution pattern for an edema magnitude of $\Delta=0.38$ and the corresponding model assessment parameters are referred for the rest of the manuscript as the mean edema magnitude resolution pattern.

Table 1. Material properties used for the modeling of the pubic organs of interest for Test 1.

\begin{tabular}{|c|c|c|c|c|}
\hline Domain & Young Modulus (MPa) & Poisson ratio & Porosity & $\begin{array}{l}\text { Permeability } \\
\left(\mathrm{mm}^{4} \mathrm{~N}^{-1} \mathrm{~s}^{-1}\right)\end{array}$ \\
\hline Prostate & $0.01-0.1^{\mathrm{a}}$ & $0.1-0.45^{\mathrm{a}}$ & 0.5 & $3.177 \mathrm{e}-05$ \\
\hline Bladder \& Rectum ${ }^{\mathrm{b}}$ & $0.015 \mathrm{MPa}$ & 0.49 & - & - \\
\hline Soft Tissue & $0.001 \mathrm{MPa}$ & 0.35 & - & - \\
\hline
\end{tabular}

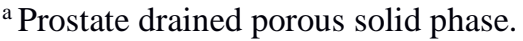

${ }^{\mathrm{b}}$ The Young modulus and Poisson ratio of the rectum and the bladder were converted to the $\mathrm{c} 1$, $\mathrm{c} 2$ Mooney-Rivlin parameters and bulk modulus.

Table 2. Computational scenarios for the investigation of the edema resolution pattern changes.

Variable Tested values

Edema magnitude $-\Delta^{\mathrm{a}} \quad 0.1,0.2,0.38,0.65$


a The edema magnitude $(\Delta)$ represents the percentage prostate volume change in decimal notation $(0.1 \rightarrow 10 \%$, $0.2 \rightarrow 20 \%$, and so on).

${ }^{\mathrm{b}}$ The rest elastic parameters were set according to table 1 and $\Delta$ was set at 0.38 for all cases.

\subsection{Post-implant dosimetry simulation}

For an accurate simulation of the post-implant dosimetry, the GGEMS-Brachy MC framework (Lemaréchal et al 2015) was used, considering the inter-seed photon interactions as well as the patientspecific tissue heterogeneities at the voxel level based on patient-specific 3D CT images. This framework developed using CUDA (Kirk 2007) enables an accurate and rapid MC simulation of the dose deposited in voxelized volumes. In GGEMS-Brachy materials from CT images are derived by transforming Hounsfield units into materials through density conversion given by Schneider et al (2000). Material mixtures and their physical properties are built using the Geant 4 materials library.

In order to integrate the modeled edema dynamics in the dosimetry simulation the computed deformation map obtained using the model's tetrahedral mesh has to be applied on the original voxelized volume (patient's pre-operative CT volume). For this step, the resampling method for interactive deformation of volumetric models previously proposed by Aguilera et al (2015) was used. This resampling method was modified to apply the model's deformation map on the initial brachytherapy seeds' positions. The brachytherapy seeds' movement (translation, no rotation) was assumed to depend exclusively on the deformation of the prostate, while any contribution to the prostate deformation from the seeds' geometry and material properties were not taken into consideration. Finally, this resampling approach was implemented using CUDA, allowing the application of the deformation map of a coarse FE mesh consisting of 3200 nodes on a voxelized volume in $23.9 \mathrm{~ms}$.

The impact of the edema model on post-implant dosimetry was investigated by comparing several dose metrics between Day1 and Day30 dosimetry similarly to previous studies (Chira et al 2013, Tejwani et al 2012). The original pre-implant CT volume of each patient and the seeds' positions, chosen in order to ensure good D90 coverage on the prostate, were used for the Day30 conventional dosimetry. For the Day 1 dosimetry simulation the model's deformation during the maximum edema magnitude state was applied both on the initial CT volume and seeds' positions. Additionally, the Day30 "dynamic dosimetry" was calculated considering the dynamic deformation of the CT volume and seeds' positions during the complete edema resolution pattern with a step size of 1 day between each deformation state for the first 30 days after the operation. Day1 dosimetry, Day30 conventional dosimetry and Day30 "dynamic dosimetry" were calculated for all the simulated edema patterns summarized previously in table 2 . All the dosimetry calculations were based on $5 \times 10^{8}$ emitted particles per simulation with an average dose uncertainty of $2 \%$ in the prostate, using a full model of 125I seeds (STM1251, Bard Medical Division, Covington, GA, USA), commonly employedin prostate cancer brachytherapy, and previously modeled in Lemaréchal et al (2015) based on the geometry and composition described in Kirov and Williamson (2001). In addition, the use of a previously proposed hybrid navigator (Bert et al 2016) allowed to consider several seeds close together within the same voxel as well as considering inter-seed interactions as previously described in Lemaréchal et al (2015).

Finally, in order to quantify the observed dose differences and potential impact, the V90 prostate (prostate volume receiving the $90 \%$ of $145 \mathrm{~Gy}$ ), D90 prostate, D10 urethra, D2cc rectum and the D10 pubic bones' 
dose metrics were computed for all the edema scenarios for Day1 and Day30 (conventional and dynamic) dosimetry.

\section{Results}

\subsection{Biomechanical model}

The mechanical properties of the proposed biomechanical model used in the computation of the mean edema magnitude resolution pattern, were manually adjusted for all patient datasets to fit the mean edema magnitude values measured in the case-study of Tejwani et al (2012) for three control time points (Day1, Day9, and Day30) after the operation (see table 3).

Table 3. Prostate mechanical properties after model assessment ${ }^{\mathrm{a}}$.

\begin{tabular}{lcccc}
\hline Domain & $\begin{array}{c}\text { Young Modulus } \\
(\mathrm{MPa})\end{array}$ & Poisson's ratio & Porosity & Permeability \\
\hline Prostate & $\mathbf{0 . 0 5}$ & $\mathbf{0 . 4}$ & $\mathbf{0 . 5}$ & $\mathbf{3 . 1 7 7 e - 0 5}$ \\
\hline $\begin{array}{l}\text { a These mechanical properties were shown to have the optimal fitting with the reference } \\
\text { ground truth measurements of Tejwani } \text { et al (2012) used in this study. }\end{array}$
\end{tabular}

For these selected parameters the model fitted well with the edema magnitude mean values measured at the control time points of the reference study (Tejwani et al 2012) considered as ground truth. The computed edema resolution pattern and the calibration values of the edema mean magnitude values at the control time points of the ground truth reference are shown in figure 2.

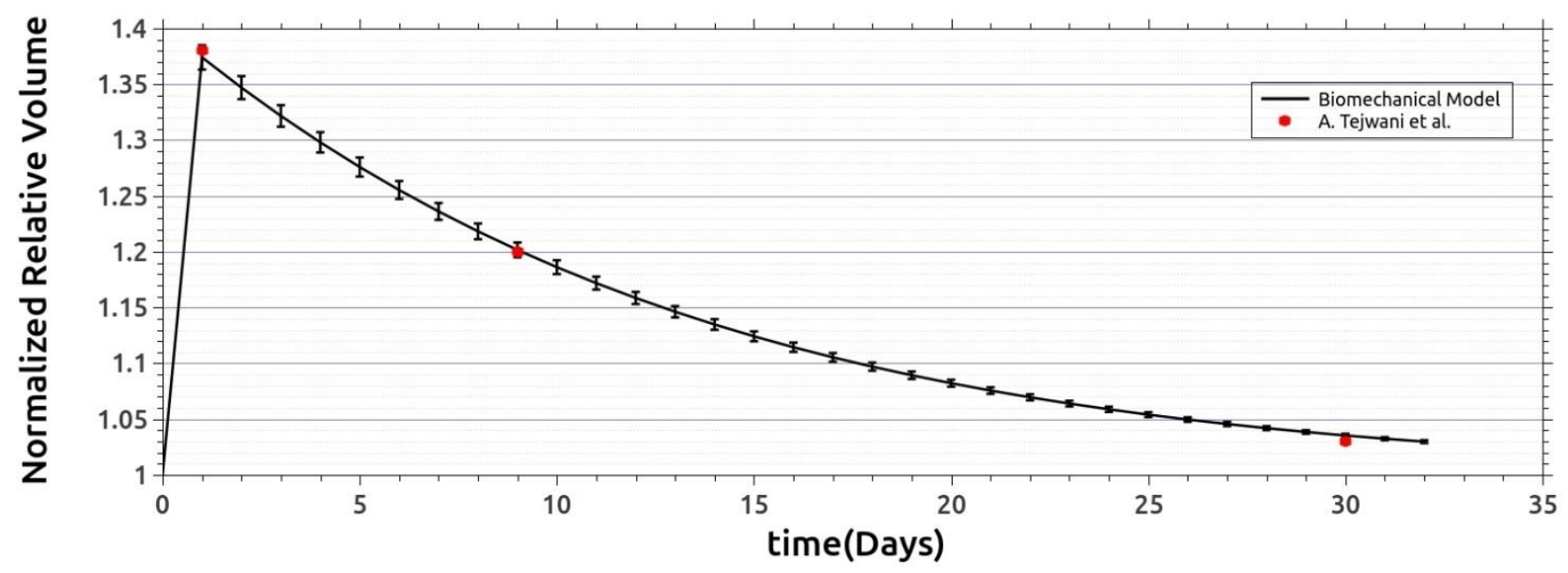

Figure 2. The solid continuous curve corresponds to the mean edema resolution pattern calculated for the 15 available patient datasets. The error bars correspond to the standard deviation (SD) considering the 15 patients and the red points correspond to the mean edema magnitude values as measured by Tejwani et al (2012). 
The dependence of the edema parameters (magnitude, half-life) on the prostate mechanical properties was studied with a series of computational tests, considering a Poisson ratio in the range of 0.1 to 0.45 and a Young modulus in the range of $0.01 \mathrm{MPa}$ to $0.1 \mathrm{MPa}$. The relationship of the edema parameters with the mechanical properties of the prostate are shown in figure 3. The proposed biomechanical model led to a higher dependence of the edema parameters on the Young modulus compared to the Poisson ratio. Prostate Young modulus, as measured for example by in-vivo shear wave elastography (Barr et al 2012), have shown high variability amongst cancer patients depending on the overall patient anatomy and tissue constitution (e.g. cancer extent or tissue inflammation). For these reasons only the impact of the prostate stiffness (Young modulus) in the post-treatment dosimetry was investigated, in addition to the impact of the edema magnitude. Figure 4 shows the different computed edema resolution patterns used in the following posttreatment dosimetry simulations.

a.

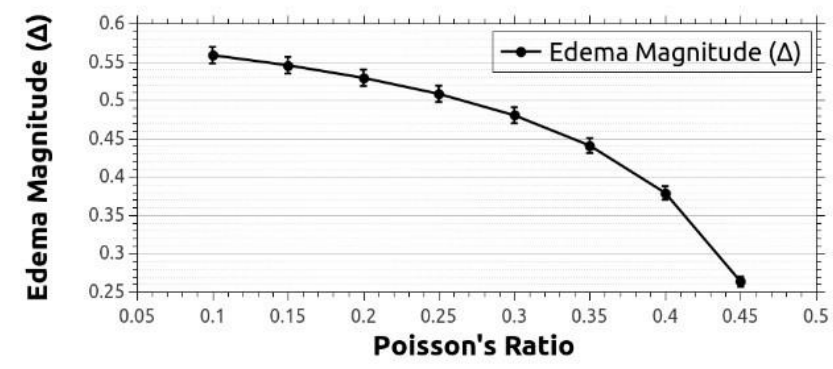

c.

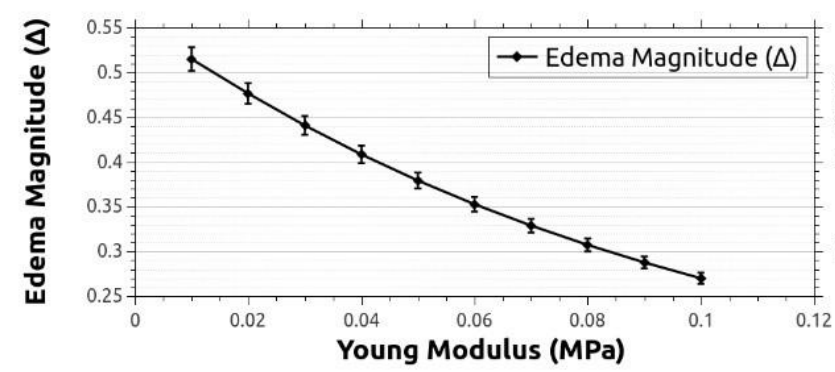

b.

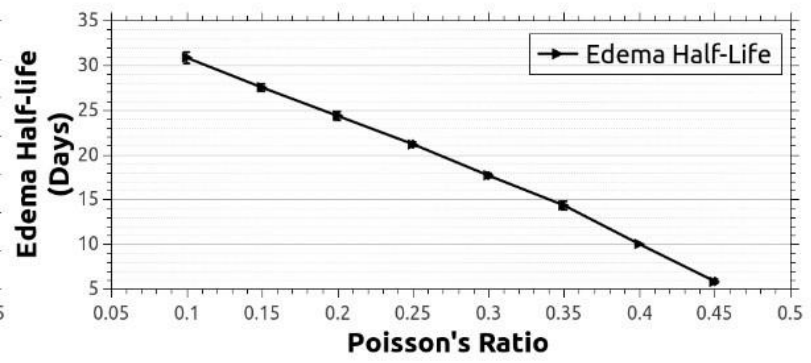

d.

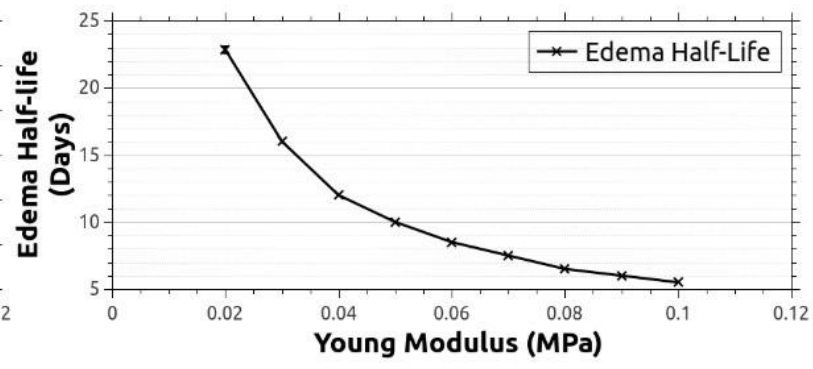

Figure 3. Relationship between the edema parameters and the prostate mechanical properties, (a). Edema magnitude dependence on the Poisson's ratio; relationship between (b). the edema half-life and the Poisson's ratio; (c). The edema half-life with the Young modulus; and (d). The edema magnitude with the Young modulus. In all the cases shown above all the other mechanical properties were those defined in table 1. The error bars show the S.D amongst the 15 patients' dataset. 


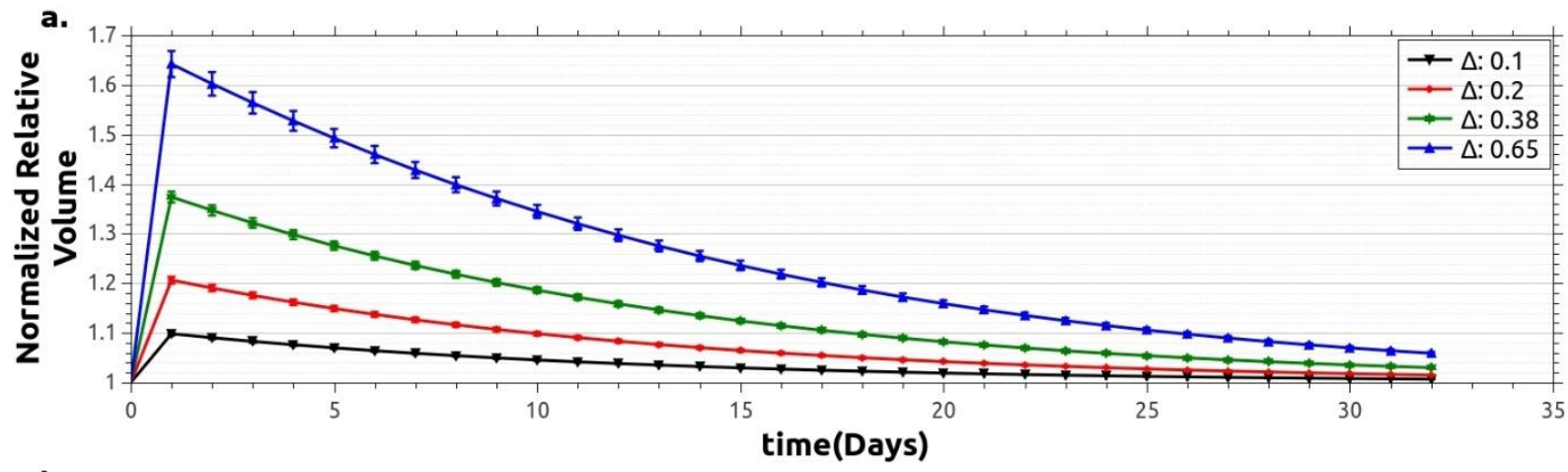

b.

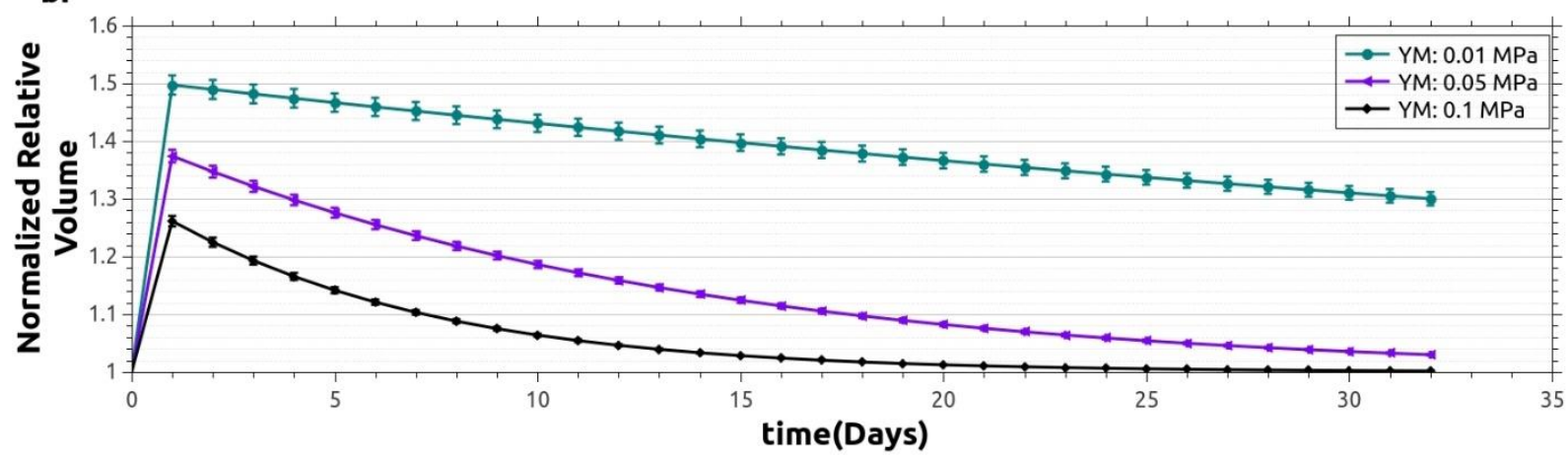

Figure 4. In figure 4(a) the edema resolution patterns for 4 different edema magnitudes at Day1 and Young Modulus 0.05 MPa are shown. Figure 4(b) shows the edema resolution patterns for the three considered Young Modulus scenarios with edema magnitude 0.38 . The error bars in both plots show the SD amongst the fifteen patient datasets.

As shown in figure 4 the prostate stiffness variance affects significantly the overall edema resolution pattern. A very elastic prostate (YM: $0.01 \mathrm{MPa}$ ) exhibits a nearly linear edema resolution pattern, while the variation of the edema maximum magnitude with constant mechanical properties doesn't affect the edema resolution pattern.

\subsection{Post-implant dosimetry simulation}

For all dosimetry simulations, the resampling method for the deformation of volumetric models was applied on the initial CT dataset and seeds' locations. Deformed CT volumes and seeds' locations were generated for each patient and each state of the biomechanical model to take into account the prostate volume changes during the first thirty days after the operation. Figures 5 and 6 show the application of the deformation on a typical patient CT volume and the corresponding seeds displacements respectively. 

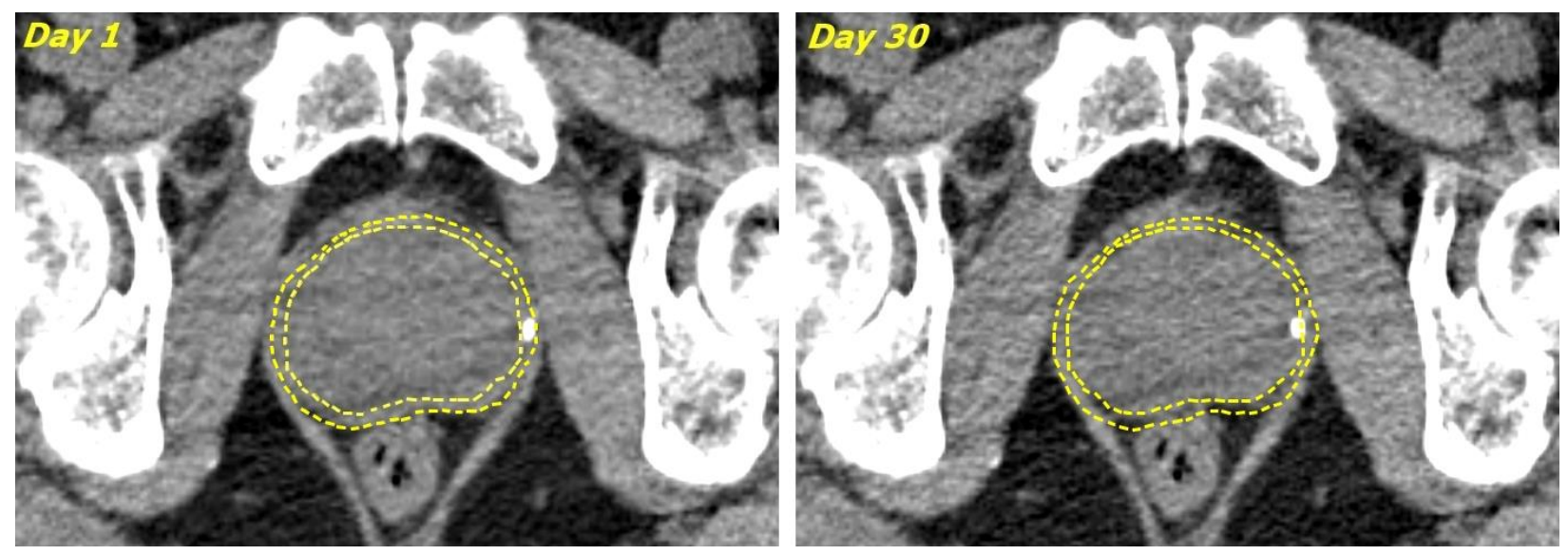

Figure 5. CT slices of the pubic region at Day1 (maximum deformation applied) and Day30. The inner and outer dashed-lines represent the Day30 and Day1 prostate contours respectively.

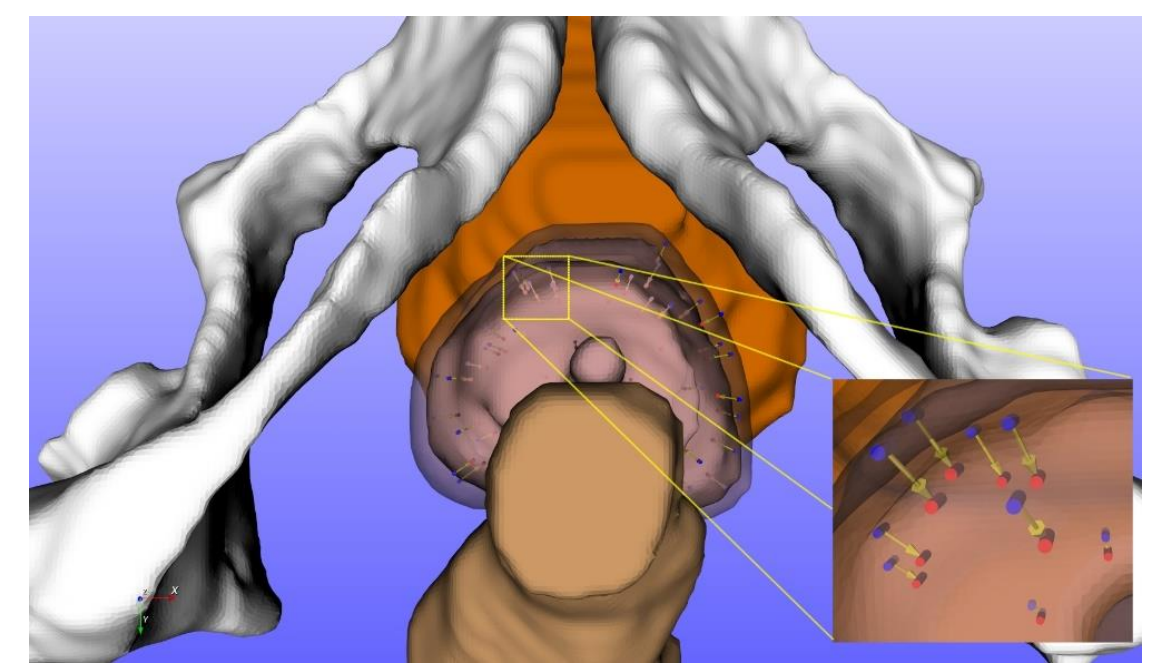

Figure 6. A super-imposed 3D representation of the pubic organs of interest. The prostate deformation and seeds' displacements between Day1 and Day30 is shown. The zoomed inset shows the displacement of the seeds from their position at Day1 (blue) to their final position at Day30 (red).

The comparison of Day1 and Day30 dosimetry simulations for the different computed edema resolution patterns highlights the overall underestimation of the actual dose deposited if Day1 dosimetry is performed without considering the edema resolution mechanism, in accordance with previous studies (Chira et al 2013, Tejwani et al 2012). All the patient datasets used in our study showed an increase in the dose differences between the Day1 and Day30 dosimetry which was proportional to the increase of the maximum edema magnitude $(\Delta)$ while the mechanical properties were considered constant. In contrast, taking into consideration the variation of the prostate stiffness, the dosimetric difference between Day1 and Day30 dosimetry was smaller for a highly elastic prostate (YM of $0.01 \mathrm{MPa}$ ) compared to a stiffer prostate (YM of $0.1 \mathrm{MPa}$ ) due to the nearly linear edema resolution pattern shown in the former scenario and the associated large edema half-life. Additionally, the comparison between the conventional and the dynamic Day30 dosimetry revealed an overestimation of the hot spots measured by the urethra D10 metric in the former 
case. The relative differences of the dose metrics for all the computed edema resolution patterns are shown in tables 4 and 5.

Table 4. Mean relative changes for the dose metrics of interest for the 15 patients' dataset between Day 1 and the conventional and/or dynamic Day30 dosimetry for the different computed edema magnitude scenarios.

\begin{tabular}{|c|c|c|c|}
\hline $\begin{array}{l}\text { Edema Magnitude } \\
\text { Scenarios }\end{array}$ & Dose Metric & $\begin{array}{c}\text { Day30 Dynamic } \\
\text { Relative Change }(\%) \pm \\
{[\mathrm{SD}]}\end{array}$ & $\begin{array}{c}\text { Day30 Conventional } \\
\text { Relative Change }(\%) \pm \\
{[\mathrm{SD}]}\end{array}$ \\
\hline \multirow{5}{*}{$\Delta: 0.1$} & Prostate V90 & $7.7 \pm[3.7]$ & $8.9 \pm[4.6]$ \\
\hline & Prostate D90 & $6.2 \pm[1.1]$ & $5.9 \pm[1.4]$ \\
\hline & Urethra D10 & $4.2 \pm[1.5]$ & $4.9 \pm[1.5]$ \\
\hline & Rectum D2cc & $-1.0 \pm[0.7]$ & $-0.6 \pm[0.8]$ \\
\hline & P. Bones D10 & $-1.3 \pm[0.3]$ & $-1.2 \pm[0.4]$ \\
\hline \multirow{5}{*}{$\Delta: 0.2$} & Prostate V90 & $19.0 \pm[3.4]$ & $21.9 \pm[4.7]$ \\
\hline & Prostate D90 & $13.6 \pm[1.5]$ & $13.4 \pm[4.1]$ \\
\hline & Urethra D10 & $10.5 \pm[3.4]$ & $12.2 \pm[4.2]$ \\
\hline & Rectum D2cc & $4.1 \pm[1.5]$ & $4.4 \pm[3.8]$ \\
\hline & P. Bones D10 & $-2.7 \pm[0.3]$ & $-2.9 \pm[0.8]$ \\
\hline \multirow{5}{*}{$\Delta: 0.38$} & Prostate V90 & $44.8 \pm[6.0]$ & $50.5 \pm[6.1]$ \\
\hline & Prostate D90 & $22.0 \pm[1.9]$ & $22.2 \pm[2.6]$ \\
\hline & Urethra D10 & $19.2 \pm[2.1]$ & $21.8 \pm[2.1]$ \\
\hline & Rectum D2cc & $9.3 \pm[2.5]$ & $9.4 \pm[3.1]$ \\
\hline & P. Bones D10 & $-4.4 \pm[0.4]$ & $-4.8 \pm[0.6]$ \\
\hline \multirow{2}{*}{$\Delta: 0.65$} & Prostate V90 & $80.1 \pm[18.4]$ & $96.7 \pm[20.9]$ \\
\hline & Prostate D90 & $35.9 \pm[5.0]$ & $37.3 \pm[6.6]$ \\
\hline
\end{tabular}




$\begin{array}{ccc}\text { Urethra D10 } & 31.2 \pm[1.9] & 36.3 \pm[5.6] \\ \text { Rectum D2cc } & 20.4 \pm[2.9] & 20.0 \pm[4.1] \\ \text { P. Bones D10 } & -6.2 \pm[0.6] & -7.0 \pm[1.1]\end{array}$

Table 5. Mean relative changes for the dose metrics of interest for the 15 patients' dataset between Day 1 and conventional/dynamic Day30 dosimetry for the different computed prostate young modulus (stiffness) scenarios considered.

\begin{tabular}{|c|c|c|c|}
\hline $\begin{array}{c}\text { Stiffness (Young Modulus) } \\
\text { Scenarios }\end{array}$ & Dose Metric & $\begin{array}{c}\text { Day30 Dynamic } \\
\text { Relative Change }(\%) \pm \\
{[\mathrm{SD}]}\end{array}$ & $\begin{array}{c}\text { Day30 Conventional } \\
\text { Relative Change }(\%) \pm \\
{[\mathrm{SD}]}\end{array}$ \\
\hline \multirow{5}{*}{ YM: 0.01} & Prostate V90 & $20.8 \pm[5.5]$ & $26.1 \pm[6.4]$ \\
\hline & Prostate D90 & $10.2 \pm[1.7]$ & $10.4 \pm[2.1]$ \\
\hline & Urethra D10 & $7.6 \pm[1.4]$ & $9.3 \pm[1.6]$ \\
\hline & Rectum D2cc & $6.2 \pm[1.0]$ & $6.3 \pm[1.4]$ \\
\hline & P. Bones D10 & $-1.8 \pm[0.3]$ & $-2.2 \pm[0.5]$ \\
\hline \multirow{5}{*}{ YM: 0.05} & Prostate V90 & $44.8 \pm[6.0]$ & $50.5 \pm[6.1]$ \\
\hline & Prostate D90 & $22.0 \pm[1.9]$ & $22.2 \pm[2.6]$ \\
\hline & Urethra D10 & $19.2 \pm[2.1]$ & $21.8 \pm[2.1]$ \\
\hline & Rectum D2cc & $9.3 \pm[2.5]$ & $9.4 \pm[3.1]$ \\
\hline & P. Bones D10 & $-4.4 \pm[0.4]$ & $-4.8 \pm[0.6]$ \\
\hline \multirow{5}{*}{ YM: 0.1} & Prostate V90 & $31.4 \pm[3.5]$ & $34.3 \pm[4.7]$ \\
\hline & Prostate D90 & $19.2 \pm[0.6]$ & $19.0 \pm[0.5]$ \\
\hline & Urethra D10 & $18.9 \pm[6.2]$ & $20.6 \pm[6.1]$ \\
\hline & Rectum D2cc & $4.2 \pm[2.5]$ & $4.3 \pm[2.8]$ \\
\hline & P. Bones D10 & $-4.2 \pm[0.3]$ & $-4.2 \pm[0.4]$ \\
\hline
\end{tabular}


The deposited dose difference to the prostate ranges between $6.21 \%$ and $35.87 \%$ considering the edema dynamics (see table 4, column 3) for 0.1 and 0.65 edema magnitude values respectively. Likewise, significant dose differences were measured in the urethra ranging between $4.20 \%$ and $31.18 \%$. In addition, dose differences are shown to depend on the prostate stiffness. The lower dose difference between Day 1 and Day30 dosimetry was measured for a prostate Young Modulus of $0.01 \mathrm{MPa}$ and was $10.17 \%$ and $7.56 \%$ for the prostate and the urethra respectively, taking into consideration the edema dynamics. The higher dose difference of $19.21 \%$ and $18.85 \%$, for the prostate and the urethra respectively, was measured for a Young Modulus of $0.1 \mathrm{MPa}$ (see table 5, column 3).

In all the tested scenarios the application of the edema biomechanical model led to an overall increase in the D90 coverage of the prostate and an increase in the dose deposited in the central region of the prostate, where the urethra is located, due to the resolution of the edema and the induced displacements of the brachytherapy seeds. These findings are displayed for the case of an average edema magnitude of $\Delta=0.38$ in terms of DVH and isodoses' distribution for a representative patient in figures 7 and 8 respectively.

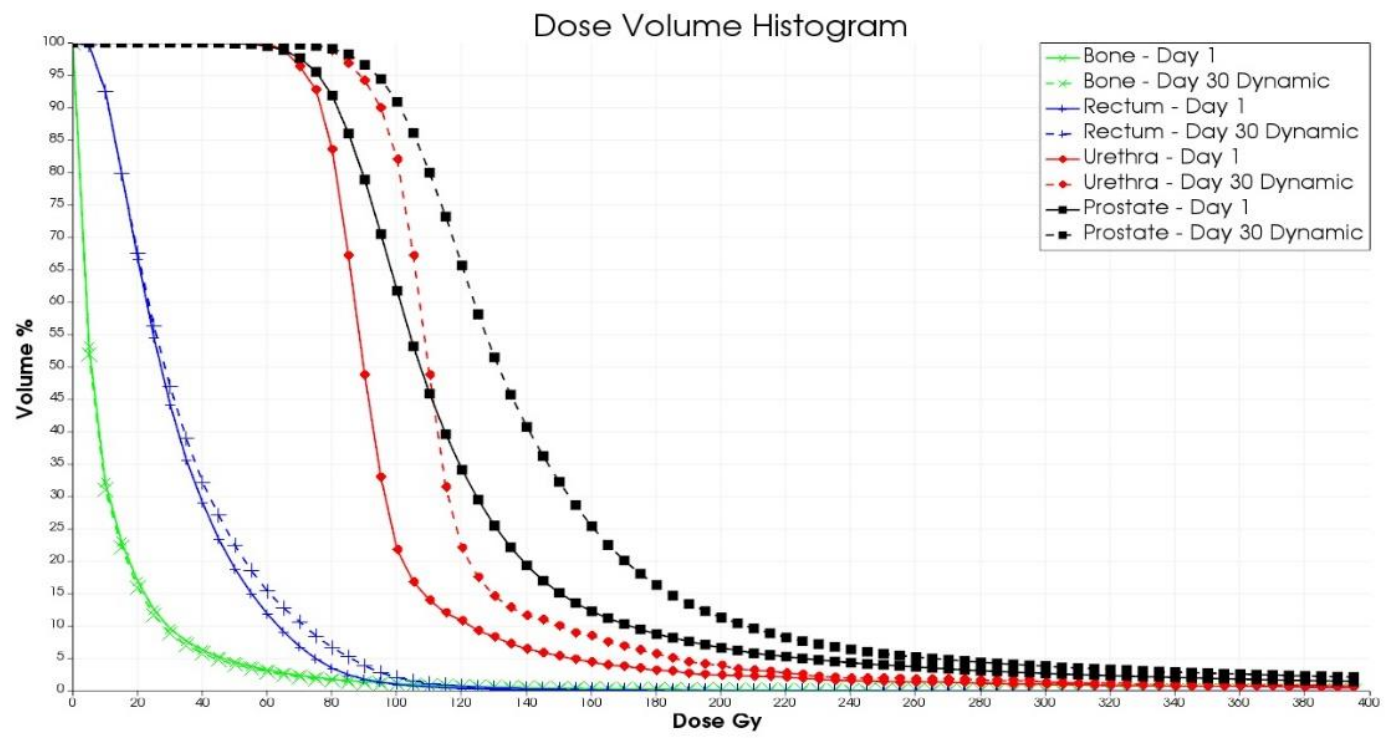

Figure 7. DVH representation of the dose deposited in the case of $\Delta=0.38$ for a representative patient. The dose deposited in the prostate and the OAR is increased when the edema resolution mechanism is considered due to the prostate contraction and the relative displacements of the seeds.
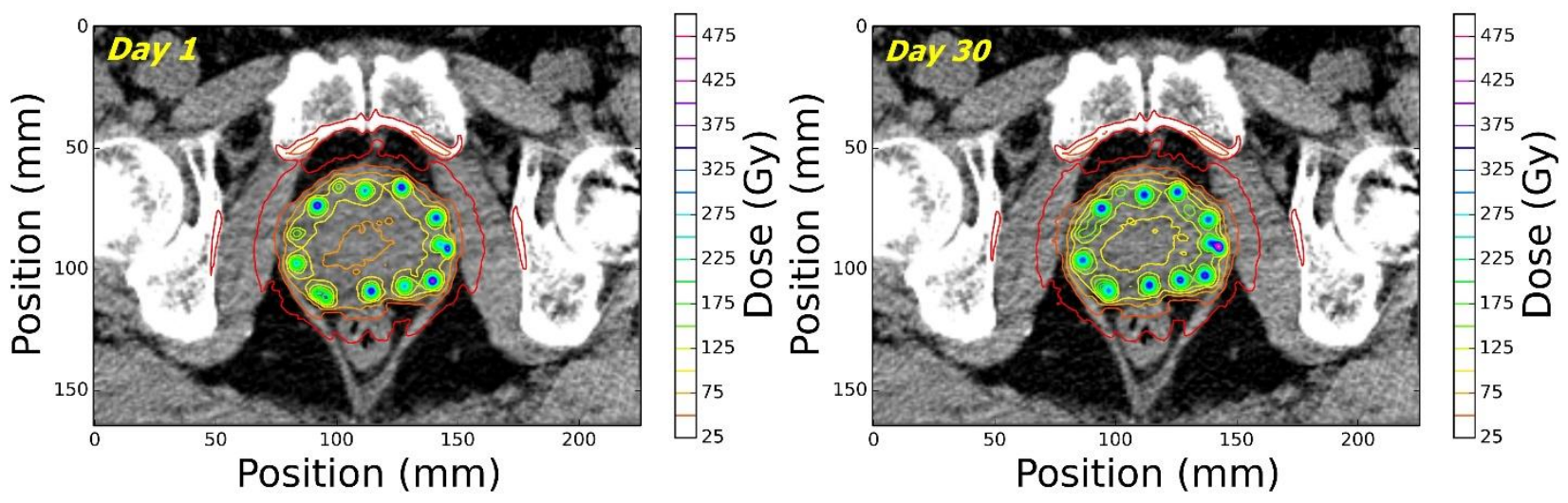
Figure 8. Lower dose deposition is recorded in the central area of the prostate, where the urethra is located, in Day1 dosimetry (a) shown by the central isodose (orange - 75 Gy) compared to the dynamic Day30 dosimetry (b) where higher dose is shown by the central isodose (yellow - $100 \mathrm{~Gy}$ ).

\section{Discussion}

Edema is an important error-inducing factor during dosimetric estimations. Big dosimetric diversions can occur depending on the edema magnitude and half-life. Previously developed mathematical models aim at correcting the dosimetric measurements applying global correction factors (Tejwani et al 2012). These correction factors are based on mean edema magnitude and half-life values. The big diversion of edema characteristics amongst patients is not considered in these models and therefore their application is limited.

The proposed biomechanical model in this study was able to successfully simulate the exponential edema resolution pattern reported in previous studies (Tejwani et al 2012, Waterman et al 1998). For the mean edema resolution pattern $(\Delta=0.38)$, a maximum uncertainty of $1.3 \%$ between the reported values in Tejwani et al (2012) at the control points (Day 1, Day 9 and Day 30) and the simulated values using the presented biomechanical model, was measured for the used dataset of 15 patients. The prostate volume changes were associated with an excessive fluid build-up, due to the occurring inflammation and the tissue mechanical properties, considering the biphasic constitution of the prostate. In contrast, with the currently available mathematical models, proposed to date, we have shown that the edema resolution pattern is sensitive to patient-specific variable mechanical properties. The contrast between the linear edema resolution pattern described by R. Sloboda et al (2010) and the exponential resolution pattern shown in previous studies can be associated with the possible prostate stiffness variance in the patient population samples used in these studies. As it was shown by our proposed model, a stiff prostate demonstrates exponential resolution pattern while with a "softer" prostate the resolution pattern may become linear. The dose differences presented in this study for different prostate Young Modulus values (see table 5) can be attributed to such edema resolution pattern differences. Therefore our study suggests that it is essential to have an a priori knowledge of the elastic properties of the prostate in order to estimate the patient specific edema resolution pattern. Ultrasound elastography (Barr et al 2012) is an appealing modality for the in vivo acquisition of the prostate elastic parameters. Our study suggests that its incorporation in the brachytherapy procedure could be beneficial for the prediction and optimization of the treatment's outcome. Additionally, in order to accurately predict the extent of the edema, further research on the brachytherapy procedure related inflammation is required. In our study a simple linear model of the external fluid pressure due to inflammation was sufficient to simulate the desired edema extent. However, a fully predictive patientspecific biomechanical model may be also possible by incorporating a more realistic inflammation mechanism in our presented model.

Furthermore, this model highlighted the divergence in post-operative dosimetry depending on the edema magnitude and resolution pattern (related edema half-life). The $11.7 \%$ increase in the prostate D90 reported by Chira et al (2013) is within the range predicted by the proposed model, with a D90 increase between $6.2 \%$ and $13.6 \%$ for an edema magnitude of between $10 \%-20 \%(\Delta=0.1-0.2)$. This has to be considered within the context of a $2 \%$ uncertainty in the prostate of our Monte Carlo based dosimetric calculations in combination with the ultrasound resolution induced error in prostate volume extraction, in addition to the lack of prostate volume measurements after the operation in the study of Chira et al (2013). In addition, given that there is no evidence that the edema occurs only during the operation, it is also possible that the edema magnitude considered in this study was underestimated.

Relatively to other parameters that may influence dosimetric accuracy in LDR brachytherapy, the prostate D90 increase of between $6.2 \%-13.6 \%$ as a result of modeling the edema effects is larger than the average of $8.7 \%$ and $3.5 \%$ differences previously reported between the AAPM Task Group 43 (TG43)/Monte Carlo based dosimetry calculations and the use of accurate prostate calcifications material definition, respectively (Lemarechal et al 2015). Within the same comparative context, a more significant impact in the dose to OAR can be seen by accounting for the edema effects, such as the urethra D10 dose increase of approximately $20 \%$ relative to $2 \%$ differences between the use of the TG43 and MC based dosimetry calculations. 
The divergence of the Day30 increase in dose metrics between the different edema scenarios shows that a global correction factor of the Day1 dosimetry as proposed by Tejwani et al (2012) cannot be considered as an accurate prediction of the actual dose delivered in the prostate and the OAR. While Day30 dosimetry can evaluate the quality of the brachytherapy procedure, assuming that the edema has resolved by then, it cannot account for the dynamic volume changes and corresponding seed displacements between Day 1 and Day30. The dynamic dosimetry algorithm proposed by Leclerc et al (2006) has shown average dose differences of $20 \mathrm{~Gy}$ in prostate D90 between Day1 and Day30 dosimetry. Similarly, our study shows that dynamic monitoring of volume changes and seeds' displacements lead to an increase in the prostate D90 of $22 \%$ but also in the urethra dose hot spots, as described by the urethra D10 of $19.2 \%$, for an average edema magnitude $(\Delta=0.38)$ between Day1 and dynamic Day30 dosimetry. The higher difference between dynamic Day30 and conventional Day30 dosimetry observed, was 5.12\% for the case of the higher edema magnitude scenario considered $(\Delta=0.65)$. This has to be considered only as an indication of the potential differences given the small number of patients considered in this study. A bigger population will be clearly necessary to establish the range of expected dosimetric differences and further validate our findings.

A limitation of the proposed mesh based deformable model is the lack of multi-resolution capabilities without an associated increase in the overall computational times. For example, in the case of large edema magnitude scenarios, the quality of the geometry discretization (tetrahedral mesh) using low quality tetrahedra can lead to higher inaccuracies in regions undergoing large deformations. If one was to use finer tetrahedra throughout the prostate volume, to account more accurately for such large deformations the overall computational times would become prohibitive for clinical use. However, a multi-resolution model would facilitate an accurate modeling of interactions and movement of fine structures, such as seeds and calcifications, within the overall prostate volume. Alternative modeling approaches such as the smoothed finite element method (Liu et al 2007) or mesh-free methods (Horton et al 2010) could possibly be used to avoid these limitations. Such models can also be used in other locally relevant phenomena such as the interactions between prostate tissue and the needles used to insert the radioactive seeds for navigation purposes.

\section{Conclusions and future work}

The first contribution of the presented study is the development of a biomechanical model capable of incorporating patient-specific mechanical properties in the prostate edema resolution pattern computation. The second contribution is the application of the computed deformation map given by the model's mesh topology determined using the original patient CT volume. To our knowledge, this is the first time a dynamic Monte Carlo simulation on deformable voxelized datasets for prostate LDR brachytherapy has been performed. This allowed us to consider the dynamic changes of the patient's anatomy and the seed locations in the dose distribution calculations, a first step towards a truly dynamic dosimetry in LDR prostate brachytherapy.

In the future and in order to improve the proposed biomechanical model we plan to incorporate localized edema effects considering the seeds' activity contribution in the extent of the edema (Tejwani et al 2012), replacing the currently used global edema assumptions. In addition, a more accurate inflammation mechanism will be developed aiming towards a predictive model, which will be subsequently introduced within a novel brachytherapy treatment planning framework, incorporating the use of ultrasound based elastography, able to predict the dynamic dose distribution in the prostate and OAR considering the edema dynamics and patient specific prostate tissue properties.

\section{Acknowledgements}

This work was partly supported by the French Brittany Region and by the French ANR within the Investissements d'Avenir program (Labex CAMI) under reference ANR-11-LABX-0004 (Integrated project CAPRI) and through FOCUS project (ANR-16-CE19-0011). 


\section{References}

Aguilera, A. R., Salas, A. L., Perandrés, D. M., \& Otaduy, M. A. (2015). A parallel resampling method for interactive deformation of volumetric models. Computers \& Graphics, 53, 147-155.

Ahn, B. M., Kim, J., Ian, L., Rha, K. H., \& Kim, H. J. (2010). Mechanical property characterization of prostate cancer using a minimally motorized indenter in an ex vivo indentation experiment. Urology, 76(4), 1007-1011.

Barr, Richard G., Richard Memo, and Carl R. Schaub. "Shear wave ultrasound elastography of the prostate: initial results." Ultrasound quarterly 28.1 (2012): 13-20.

Beaulieu L, Carlsson Tedgren A, Carrier J-F, Davis S D, Mourtada F, Rivard M J, Thomson R M, Verhaegen F, Wareing T A and Williamson J F 2012 Report of the task group 186 on modelbased dose calculation methods in brachytherapy beyond the tg- 43 formalism: current status and recommendations for clinical implementation Med. Phys. 39 6208-36.

Bert, Julien, Yannick Lemaréchal, and Dimitris Visvikis. "New hybrid voxelized/analytical primitive in Monte Carlo simulations for medical applications." Physics in medicine and biology 61.9 (2016): 3347.

Chen, Z., Yue, N., Wang, X., Roberts, K. B., Peschel, R., \& Nath, R. (2000). Dosimetric effects of edema in permanent prostate seed implants: a rigorous solution. International Journal of Radiation Oncology* Biology* Physics, 47(5), 1405-1419.

Chira, C., Delouya, G., Larrivée, S., Carrier, J. F., \& Taussky, D. (2013). Prostate volume changes during permanent seed brachytherapy: an analysis of intra-operative variations, predictive factors and clinical implication. Radiation Oncology, 8(1), 177.

Davies, B. L., Harris, S. J., \& Dibble, E. (2004). Brachytherapy - an example of a urological minimally invasive robotic procedure. The International Journal of Medical Robotics and Computer Assisted Surgery, 1(1), 88-96.

Day, J., Rubin, J., Vodovotz, Y., Chow, C. C., Reynolds, A., \& Clermont, G. (2006). A reduced mathematical model of the acute inflammatory response II. Capturing scenarios of repeated endotoxin administration. Journal of theoretical biology, 242(1), 237-256.

Dogan, N., Mohideen, N., Glasgow, G. P., Keys, K., \& Flanigan, R. C. (2002). Effect of prostatic edema on CT-based postimplant dosimetry. International Journal of Radiation Oncology* Biology* Physics, 53(2), 483-489.

Fang, Q., \& Boas, D. A. (2010). ISO2Mesh: a 3D surface and volumetric mesh generator for matlab/octave.

Galbusera, F., Schmidt, H., Noailly, J., Malandrino, A., Lacroix, D., Wilke, H. J., \& Shirazi-Adl, A. (2011). Comparison of four methods to simulate swelling in poroelastic finite element models of intervertebral discs. Journal of the mechanical behavior of biomedical materials, 4(7), 1234-1241.

Haas, G. P., Delongchamps, N., Brawley, O. W., Wang, C. Y., \& de la Roza, G. (2008). The worldwide epidemiology of prostate cancer: perspectives from autopsy studies. The Canadian journal of urology, 15(1), 3866.

Haridas, Balakrishna, et al. "PelvicSim-A computational-experimental system for biomechanical evaluation of female pelvic floor organ disorders and associated minimally invasive interventions." Stud. Health Technol. Inf 119 (2006): 182-187.Horton, Ashley, et al. "A meshless Total Lagrangian explicit dynamics algorithm for surgical simulation." International Journal for Numerical Methods in Biomedical Engineering 26.8 (2010): 977 998.

Hu, Y., Morgan, D., Ahmed, H. U., Pendsé, D., Sahu, M., Allen, C. \& Barratt, D. (2008). Modelling prostate gland motion for image-guided interventions. In Biomedical Simulation (pp. 79-88). Springer Berlin Heidelberg.

Kirk, David. "NVIDIA CUDA software and GPU parallel computing architecture." ISMM. Vol. 7. 2007.

Kirov, Assen S., and Jeffrey F. Williamson. "Monte Carlo-aided dosimetry of the Source Tech Medical Model STM1251 I-125 interstitial brachytherapy source." Medical physics 28.5 (2001): 764-772.

Leclerc, Ghyslain, et al. "Prostatic edema in I125 permanent prostate implants: Dynamical dosimetry taking volume changes into account." Medical physics 33.3 (2006): 574-583.

Lee, H. P., Lin, M. C., \& Foskey, M. (2008). Physically-based validation of deformable medical image registration. In Medical Image Computing and Computer-Assisted Intervention-MICCAI 2008 (pp. 830-838). Springer Berlin Heidelberg.

Lemaréchal, Y., Bert, J., Falconnet, C., Després, P., Valeri, A., Schick, U., ... \& Visvikis, D. (2015). GGEMS-Brachy: GPU GEant4-based Monte Carlo simulation for brachytherapy applications. Physics in medicine and biology, 60(13), 4987.

Li, C., Guan, G., Zhang, F., Song, S., Wang, R. K., Huang, Z., \& Nabi, G. (2014). Quantitative elasticity measurement of urinary bladder wall using laser-induced surface acoustic waves. Biomedical optics express, 5(12), 4313-4328.

Li, X. G., von Holst, H., Ho, J., \& Kleiven, S. (2009). 3-D Finite Element Modeling of Brain Edema: Initial Studies on Intracranial Pressure Using Comsol Multiphysics. In Excerpt from the Proceedings of the COMSOL Conference. 
Liu, G. R., et al. "Theoretical aspects of the smoothed finite element method (SFEM)." International journal for numerical methods in Engineering 71.8 (2007): 902-930.

Maas, S. A., Ellis, B. J., Ateshian, G. A., \& Weiss, J. A. (2012). FEBio: finite elements for biomechanics. Journal of biomechanical engineering, 134(1), 011005.

Maas, S. A., Rawlins D., Jeffrey W., Ateshian G. (2015). FeBio 2.4 theory manual, http://febio.org/febio/febiodocumentation.

Nath, Ravinder, et al. "AAPM recommendations on dose prescription and reporting methods for permanent interstitial brachytherapy for prostate cancer: Report of Task Group 137." Medical physics 36.11 (2009): 53105322.

Rivard, M. J., Coursey, B. M., DeWerd, L. A., Hanson, W. F., Huq, M. S., Ibbott, G. S. \& Williamson, J. F. (2004). Update of AAPM Task Group No. 43 Report: A revised AAPM protocol for brachytherapy dose calculations. Medical physics, 31(3), 633-674.

Schneider, Wilfried, Thomas Bortfeld, and Wolfgang Schlegel. "Correlation between CT numbers and tissue parameters needed for Monte Carlo simulations of clinical dose distributions." Physics in medicine and biology 45.2 (2000): 459.

Schroeder, W. J., Avila, L. S., \& Hoffman, W. (2000). Visualizing with VTK: a tutorial. Computer Graphics and Applications, IEEE, 20(5), 20-27.

Sloboda, R. S., Usmani, N., Monajemi, T. T., \& Liu, D. M. (2012). Impact of edema and seed movement on the dosimetry of prostate seed implants. Journal of medical physics/Association of Medical Physicists of India, 37(2), 81.

Sloboda, R. S., Usmani, N., Pedersen, J., Murtha, A., Pervez, N., \& Yee, D. (2010). Time course of prostatic edema post permanent seed implant determined by magnetic resonance imaging. Brachytherapy, 9(4), 354-361.

Starling, E. H. (1896). On the absorption of fluids from the connective tissue spaces. The Journal of physiology, 19(4), 312-326.

Tejwani, A., Bieniek, E., Puckett, L., Lavaf, A., Guirguis, A., Bennish, A., \& Ashamalla, H. (2012). Clinical Investigations: Case series analysis of post-brachytherapy prostate edema and its relevance to post-implant dosimetry. Post-implant prostate edema and dosimetry. Journal of Contemporary Brachytherapy, 4(2), 75.

Villeneuve, Maxime, et al. "Relationship between isotope half-life and prostatic edema for optimal prostate dose coverage in permanent seed implants." Medical Physics-New York-Institute of Physics 35.5 (2008): 1970-1977.

Wang, Yi, et al. "Patient-specific Deformation Modelling via Elastography: Application to Image-guided Prostate Interventions." Nature Publishing Group. Scientific Reports 6 (2016): 27386.

Waterman, F. M., Yue, N., Corn, B. W., \& Dicker, A. P. (1998). Edema associated with I-125 or Pd-103 prostate brachytherapy and its impact on post-implant dosimetry: an analysis based on serial CT acquisition. International Journal of Radiation Oncology* Biology* Physics, 41(5), 1069-1077.

Yan, Zhennan, et al. "Modulus reconstruction from prostate ultrasound images using finite element modeling." SPIE Medical Imaging. International Society for Optics and Photonics, 2012.

Yue, N., Mori, J., Nath, R., Heron, D. E., \& Huq, M. S. (2006). External beam radiotherapy boosts to reduce the impact caused by edema in prostate permanent seed implants. Physics in medicine and biology, 51(9), 2267. 